\title{
Combined DCT and Companding for PAPR Reduction in OFDM Signals
}

\section{Zhongpeng Wang}

School of Information and Electronic Engineering, Zhejiang University of Science and Technology, Hangzhou, China. Email: wzp1966@sohu.com

Received March 21 ${ }^{\text {st }}, 2011$; revised April 12 ${ }^{\text {th }}, 2011$; accepted April 21 ${ }^{\text {st }}, 2011$.

\begin{abstract}
The high peak-to-average (PAPR) is one of the serious problems in the application of OFDM technology. The companding transform approach is a very attractive technique to reduce PAPR, but large PAPR reduction leads to a high bit error rate (BER) by the available companding transform techniques. In this paper, a joint reduction in PAPR of the OFDM signals based on combining the discrete cosine transform (DCT) with companding is proposed. In the first step of the proposed scheme, the data are transformed by a DCT into new modified data. In the second step, the proposed scheme utilizes the companding technique to further reduce the PAPR of the OFDM signal. The performance of the $P A P R$ is evaluated using a computer simulation. The simulation results indicate that the proposed scheme may obtain about 1 dB PAPR reduction compared with the conventional companding algorithm.
\end{abstract}

Keywords: Companding, DCT Transform, PAPR, OFDM

\section{Introduction}

OFDM (orthogonal-frequency-division multiplexing) is a promising technique that is able to provide high data rates over multipath fading channels. However, OFDM systems have the inherent problem of a high peak-toaverage power ratio (PAPR), which causes poor power efficiency or serious performance degradation in the transmitted signal. To reduce the PAPR, many techniques have been proposed, such as clipping, coding, partial transmit sequence (PTS), selected mapping (SLM) [1-3], nonlinear companding transforms [4,5], and Hadamard transforms [6]. These schemes are primarily signal scrambling techniques, such as PTS, and signal distortion techniques such as the clipping and companding techniques. Among those PAPR reduction methods, the simplest scheme to use is the clipping process. However, use of the clipping processing causes both in-band distortion and out-of-band distortion, and causes an increased bit error rate (BER) in the system. As an alternative approach, the companding technique shows better performance than the clipping technique, because the inverse companding transform (expanding) can be applied at the receiver end to reduce the distortion of signal. A DCT may reduce the PAPR of an OFDM signal, but does not increase the BER of system. Park et al. [6] proposed a scheme for PAPR reduction in OFDM transmission using a Hadamard transform. The proposed Hadamardtransform scheme may reduce the occurrence of the high peaks when compared the original OFDM system. The idea is to use the Hadamard transform to reduce the autocorrelation of the input sequence to reduce the peak to average power problem. In addition, it requires no side information to be transmitted to the receiver. Inspired by the literature [6,7], we propose an efficient PAPR reducing technique based on a joint companding and DCT method. The proposed scheme makes use of the character domain. The data encoded in the OFDM signal are modulated by an IFFT (inverse fast Fourier transform) after being processed with the DCT, which can reduce the PAPR of OFDM signals. Then, companding algorithm is applied further to reduce the PAPR of the OFDM signal after the IFFT operation. This scheme will be compared with the original system with companding technique for reduction PAPR.

The organization of this paper is as follow. Section 2 presents the PAPR problem of OFDM signals. Companding transform and DCT transform are introduced in section 3 and section 4. In section 5, a PAPR reduction scheme by combing companding transform and DCT transform is proposed. Simulation results and performance analysis are reported in section 6 and conclusions are presented in 7 . 


\section{PAPR Problem of OFDM Signals}

An OFDM signal consists of $N$ data symbols transmitted over $N$ distinct subcarriers. Let $\boldsymbol{X}=\left\{X_{k}, k=0,1, \cdots, N-1\right\}$ be a block of $N$ symbols formed by each symbol modulating one of a set of subcarriers $\left\{f_{k}, k=0,1, \cdots, N-1\right\}$. The $N$ subcarriers are chosen to be orthogonal, that is, $f_{k}=k \Delta f$, where $\Delta f=1 /(N T)$ and $T$ is the original symbol period. Therefore, the complex baseband OFDM signal can be written as

$$
x(t)=\frac{1}{\sqrt{N}} \sum_{k=0}^{N-1} X_{k} \mathrm{e}^{j 2 \pi f_{k} t} \quad 0 \leq t \leq N T
$$

In general, the PAPR of the OFDM signal, $x(t)$, is defined as the ratio between the maximum instantaneous power and its average power during an OFDM symbol

$$
\text { PAPR }=\frac{\max _{0 \leq t \leq N T}\left[|x(t)|^{2}\right]}{1 /(N T) \int_{0}^{N T}|x(t)|^{2} \mathrm{~d} t}
$$

Reducing $\max |x(t)|$ is the principle goal of PAPR reduction techniques. In practice, most systems deal with a discrete-time signal. Therefore, we have to sample the continuous-time signal $x(t)$.

To better approximate the PAPR of continuous-time OFDM signals, the OFDM signals samples oversampled by a factor of $L$. By sampling, $x(t)$ defined in Equation (1), at frequency $f_{s}=L / T$, where $L$ is the oversampling factor, the discrete-time OFDM symbol can be given by:

$$
x(n)=\frac{1}{\sqrt{N}} \sum_{k=0}^{N-1} X_{k} \mathrm{e}^{j \frac{2 \pi}{N L} k n} \quad 0 \leq n \leq N L-1
$$

Equation (2) can be implemented using an IFFT operation of length $(N L)$. The new input vector, $X$, is extended from the original $X$ by using the zero-padding scheme, i.e. by inserting $(L-1) N$ zeros in the middle of $X$. The PAPR computed from the $L$-oversampled time-domain OFDM signal can be defined as:

$$
\operatorname{PAPR}[x(n)]=101 \mathrm{~g} \frac{\max _{0 \leq n \leq N L-1}\left[|x(n)|^{2}\right]}{E\left[|x(n)|^{2}\right]}
$$

where the expectation is taken over all OFDM symbols.

\section{Companding Transform}

In this section, we review companding techniques [5] for the reduction of the PAPR in an OFDM signal. In this section we study a companding transform, in which compression is used at the transmit end after the IFFT process and is used expansion at the receiver end prior to the FFT (fast Fourier transform) process.

For the discrete OFDM signal given by Equation (3), the companded signal $s(n)$ can be given by:

$$
s(n)=C\{x(n)\}=\frac{v x(n)}{\ln (1+u)|x(n)|} \ln \left(1+\frac{u}{v}|x(n)|\right)
$$

where $v$ is the average amplitude of the signal and $u$ is the companding parameter. Specifically, the companding transform should satisfy the following two conditions:

$$
\begin{gathered}
E\left(|s(n)|^{2}\right) \approx E\left(|x(n)|^{2}\right) . \\
|s(n)| \geq|x(n)|, \text { for }|x(n) \leq v| ; \\
|s(n)| \leq|x(n)|, \text { for }|x(n) \geq v| .
\end{gathered}
$$

This transform reduces the PAPR of OFDM signal by amplifying the small signal and attenuating the period of high signal.

On the receiver end, the receiver signal must be expanded by the inverse companding transform before it can be sent to the FFT processing unit. The expanded signal at the receiver is

$$
y(n)=C^{-1}\{r(n)\}=\frac{v r(n)}{u|r(n)|}\left\{\exp \left[\frac{|r(n)| \ln (1+u)}{v}\right]-1\right\}
$$

\section{Discrete Cosine Transform}

Like other transforms, such as the Hadamard transform, the DCT decorrelates the data sequence. To reduce the PAPR in an OFDM signal, a DCT is applied to reduce the autocorrelation of the input sequence before the IFFT operation is applied [8]. In this section, we briefly review the DCT. The formal definition of a one-dimensional DCT of length $N$ is given by the following formula:

$$
\begin{aligned}
& X_{c}(k)=\alpha(k) \sum_{n=0}^{N-1} x(n) \cos \left[\frac{\pi(2 n+1) k}{2 N}\right], \\
& \text { for } k=0, \cdots, N-1
\end{aligned}
$$

Similarly, the inverse transformation is defined as

$$
\begin{aligned}
& x(n)=\sum_{k=0}^{N-1} \alpha(k) X_{c}(k) \cos \left[\frac{\pi(2 n+1) k}{2 N}\right], \\
& \text { for } n=0, \cdots, N-1
\end{aligned}
$$

For both Equations (10) and (11) $\alpha(k)$ is defined as

$$
\alpha(k)= \begin{cases}\frac{1}{\sqrt{N}}, & \text { for } \quad k=0 \\ \frac{2}{\sqrt{N}}, & \text { for } k \neq 0\end{cases}
$$

Equation (10) can be expressed in matrix form as: 


$$
\boldsymbol{X}_{\mathrm{c}}=\boldsymbol{C}_{N} \boldsymbol{X}
$$

where $\boldsymbol{X}_{\boldsymbol{c}}$ and $\boldsymbol{x}$ are both vectors of dimension $N \times 1$, and $C_{N}$ is a DCT matrix of dimension $N \times N$. The rows (or column) of the DCT matrix, $C_{N}$, are orthogonal matrix vectors. We can use this property of the DCT matrix and reduce the peak power of OFDM signals.

According to [9], there is a close relation between the PAPR of an OFDM signal and the aperiodic autocorrelation function (ACF) of an input vector. Assume $\rho(i)$ is the ACF of a signal vector, $X$, then:

$$
\rho(i)=\sum_{k=0}^{N-1-i} X_{k+i} X_{k}^{*} \text { for } i=0,1, \cdots, N-1
$$

where the superscript $*$ denotes the complex conjugate. Then, the PAPR of the transformed OFDM signal is bounded by [9]:

$$
\text { PAPR } \leq 1+\frac{2}{N} \sum_{i=1}^{N-1}|\rho(i)|
$$

Let $\lambda=\sum_{i=1}^{N-1}|\rho(i)|$, we found that an input vector with a lower $\lambda$ yields a signal with a lower PAPR in OFDM systems. It has been proved that if input vector passed by DCT transform before IFFT, the $\rho(i)$ and thus PAPR could be reduced [9].

\section{Proposed Scheme}

To reduce the PAPR an OFDM signal, we propose a scheme involving the combination of a companding transform and DCT. The input data stream is processed with a DCT then with an IFFT signal processing unit. A block diagram of the system is shown in Figure 1.

The key signal processing step is described as below:

Step 1: The sequence $X$ is transformed using the DCT matrix, i.e. $\boldsymbol{Y}=\boldsymbol{H} \boldsymbol{X}$.

Step 2: An IFFT(Y) is applied, yielding:

$$
y=[y(1) y(2), \cdots, y(N)]^{T} .
$$

Step 3: A companding transform is then applied to $y$,

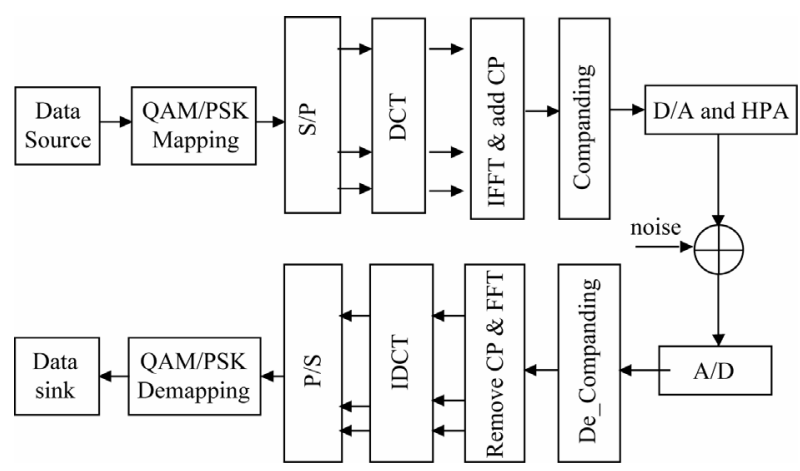

Figure 1. OFDM system block with DCT-companding. i.e. $s(n)=C\{y(n)\}$.

Step 4: An inverse companding transform is applied to the received signal, $r(n)$, i.e. $\hat{y}(n)=C^{-1}\{r(n)\}$.

Step 5: A FFT transform is applied to the signal, $\hat{y}(n)$, i.e. $\hat{\boldsymbol{Y}}=\operatorname{FFT}(\hat{\boldsymbol{y}})$, where $\hat{\boldsymbol{y}}=[\hat{y}(1) \hat{y}(2), \cdots, \hat{y}(N)]^{T}$.

Step 6: An inverse DCT transforms applied to the signal, $\hat{\boldsymbol{Y}}$, i.e. $\hat{\boldsymbol{X}}=\boldsymbol{H}^{T} \hat{\boldsymbol{Y}}$. Then, the signal, $\hat{\boldsymbol{X}}$, is demaped from the bit stream.

\section{Simulation Results}

In this section, we present the results of computer simulations used to evaluate PAPR reduction capability and BER of the proposed scheme. The channel was modeled as additive white Gaussian noise (AWGN). In the simulation, an OFDM system with a sub-carrier of $N=128,512$ and QPSK modulation was considered. We can evaluate the performance of the PAPR reduction scheme using the complementary cumulative distribution (CCDF) of the PAPR of the OFDM signal.

\subsection{CCDF Performance}

We can evaluate the performance of PAPR using the cumulative distribution of PAPR of OFDM signal. The cumulative distribution function (CDF) is one of the most regularly used parameters, which is used to measure the efficiency of and PAPR technique. The CDF of the amplitude of a signal sample is given by

$$
F(z)=1-\exp (z)
$$

However, the complementary CDF (CCDF) is used instead of CDF, which helps us to measure the probability that the PAPR of a certain data block exceeds the given threshold. The CCDF of the PAPR of the data block is desired is our case to compare outputs of various reduction techniques. This is given by

$$
P(\operatorname{PAPR}>z)=1-P(\operatorname{PAPR}>z)=1-(1-\exp (-z))^{N}
$$

Figure 2 shows the CCDF performance of a companding algorithm for PAPR reduction. The values of the companding factor, $u$, for the companding procedure of the second step were fixed to 2, 3, and 5. With this companding method, the peak power at $\mathrm{CCDF}=10^{-3}$ is reduced by $3.5 \mathrm{~dB}, 5 \mathrm{~dB}$ and $5.5 \mathrm{~dB}$ when compared with the case of original system.

Figure 3 shows the CCDF performance of the DCT scheme compared with that of the original and Hadamard transform techniques. At CCDF $=10^{-3}$, the DCT scheme reduces the PAPR by $3 \mathrm{~dB}$ over original system, but the Hadamard transform only reduced the PAPR by $1 \mathrm{~dB}$.

Figure 4 shows the CCDF performance of the proposed PAPR reduction scheme. In the simulation OFDM system, the number of sub carrier is 128 . At CCDF $=10^{-3}$, 


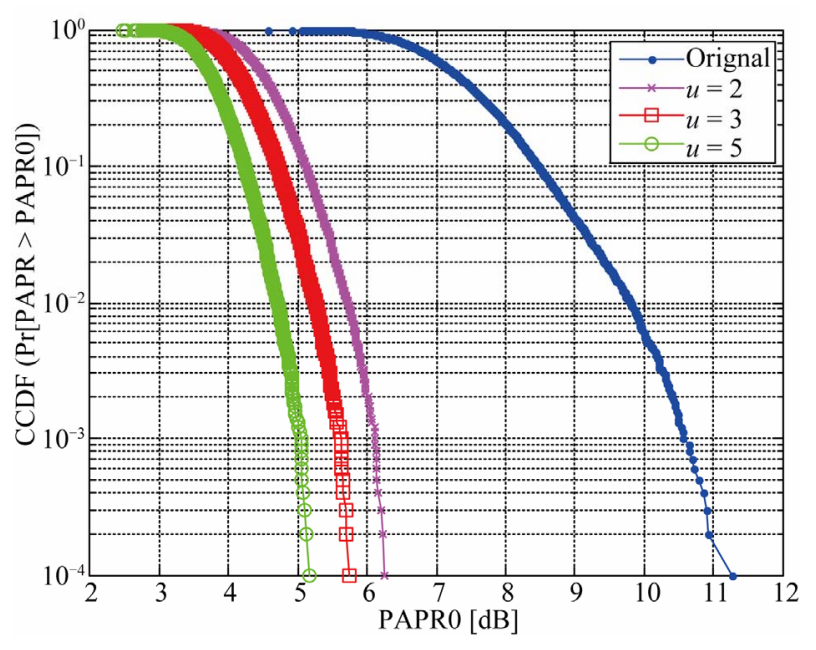

Figure 2. Comparisons of the CCDF of different companding factor $u$.

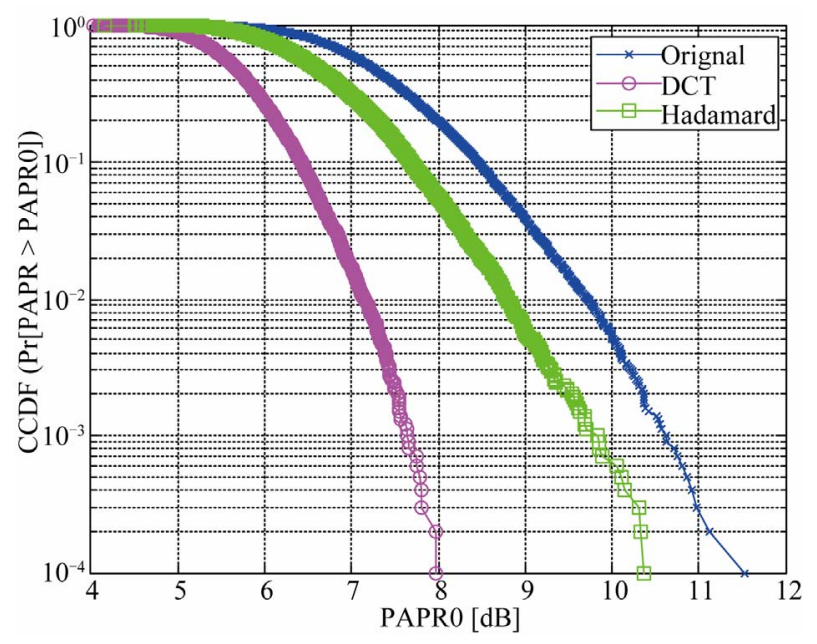

Figure 3. CCDFs of the matrix transformations.

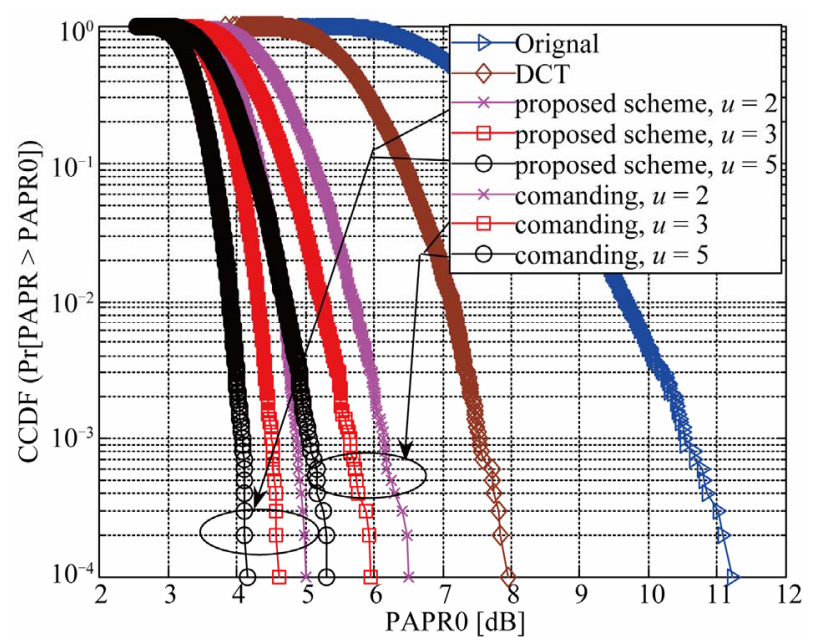

Figure 4. Comparisons of the CCDF of different PAPR reduction schemes. the proposed scheme reduces the PAPR $1 \mathrm{~dB}$ more than the companding method and reduces PAPR $2.5 \mathrm{~dB}$ more than the DCT method.

Figure 5 is the CCDF performance of proposed reduction PAPR scheme at difference subcarriers. We can see from Figure 5, the effect of difference subcarriers to PAPR performance of OFDM signals is very small.

\subsection{Analysis of Algorithm Complexity}

Compared to the ordinary companding algorithm, the computational complexity of the proposed scheme is increased because the DCT is used. However, like FFT, there are many fast methods to computer DCT. In literature [10], a fast DCT algorithm is proposed and the algorithm requires $\frac{\mathrm{N}}{2} \log _{2} \mathrm{~N}$ multiplications and $\frac{3 N}{2} \log _{2} N-N+1$ additions for $N$-length sequence. So the $N \log _{2} N$ multiplications and $2\left(\frac{3 N}{2} \log _{2} N-N+1\right)$ additions are added in proposed PAPR scheme.

\section{Conclusions}

In this paper, while taking both PAPR performance and BER performance into account, we proposed a combined DCT and companding scheme for the reduction of the PAPR of OFDM signals. The proposed scheme is composed of the DCT transform followed by the companding transform. The DCT, used in the first step, does not influence the BER. The PAPR reduction performance of the proposed scheme was evaluated using a computer simulation. The simulation results show that the PAPR reduction is improved when compared with those of a companding transform.

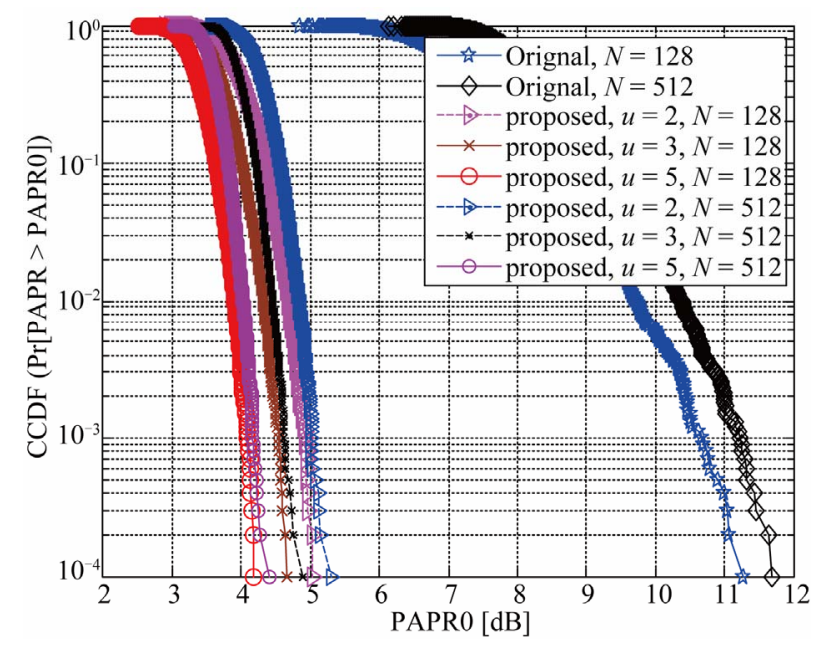

Figure 5. Comparisons of the CCDF of proposed scheme with different subcarriers. 


\section{REFERENCES}

[1] T. Jiang and Y. Imai, "An Overview: Peak-To-Average Power Ratio Reduction Techniques for OFDM Signals," IEEE Transactions on Broadcasting, Vol. 54, No. 2, 2008, pp. 257- 268. doi:10.1109/TBC.2008.915770

[2] S. H. Han and J. H. Lee, "An Overview of Peak-to-Average Power Ratio Reduction Techniques for Multicarrier Transmission," IEEE Transaction on Wireless Communication, April 2005, pp. 56-65.

[3] I. Sohn, "RBF Neural Network Based SLM Peak-toAverage Power Ratio Reduction in OFDM Systems," ETRI Journal, Vol. 29, No. 3, 2007, pp. 402-404. doi:10.4218/etrij.07.0206.0155

[4] X. B. Wang, "Reduction of Peak-to-Average Power Ratio of OFDM System Using a Companding Technique," IEEE Transaction on Broadcasting, Vol. 45, No. 3, 1999, pp. 303-307. doi:10.1109/11.796272

[5] X. Huang, J. H. Lu, J. L. Zhen, et al., "Companding Transform for Reduction in Peak-to-Average Power of OFDM Signals," IEEE Transactions on Wireless Communications, Vol. 3, No. 6, 2004, pp. 2030-2039.
doi:10.1109/TWC.2004.837619

[6] M. Park, J. Heeyong, N. Cho, D. Hong and C. Kang, "PAPR Reduction in OFDM Transmission Using Hadamard Transform," IEEE International Conference on Communications, Vol. 1, Jun 2000, pp. 430-433.

[7] J. Kim and Y. Shin, "An Effective Clipping Companding Scheme for PAPR Reduction of OFDM Signal," IEEE International Conference on Communications 2008, Beijing, 2008, pp. 668-672.

[8] X. D. Zhu, G. X. Zhu and T. Jiang, "Reducing the Peak-toAverage Power Ratio Using Unitary Matrix Transformation,' IET Communications, Vol. 3, No. 2, 2009, pp.161-171. doi:10.1049/iet-com:20080194

[9] C. Tellambura, "Upper Bound on Peak Factor of N- Multiple Carriers," Electronics Letter, Vol. 36, No. 14, 2000, pp. 1226-1228.

[10] W. J. Yuan, P. W. Hao and C. Xu, "Matrix Factorization for Fast DCT algorithms," IEEE International Conference on Acoustics, Speech and Signal Processing, 2006, pp. 948-951. 